\title{
Formation and Disordered Degradation of Neutrophil Extracellular Traps in Necrotizing Lesions of Anti-Neutrophil Cytoplasmic Antibody-Associated Vasculitis
}

\author{
Sakiko Masuda, ${ }^{* \dagger}$ Mayu Nonokawa, ${ }^{*}$ Emika Futamata, ${ }^{*}$ Yuka Nishibata, ${ }^{*}$ Sari Iwasaki, ${ }^{\ddagger}$ Takahiro Tsuji, ${ }^{\ddagger}$ Yutaka Hatanaka, \\ Daigo Nakazawa, ${ }^{\dagger}$ Satoshi Tanaka, " Utano Tomaru, "Tamihiro Kawakami, ${ }^{* *}$ Tatsuya Atsumi, ${ }^{\dagger}$ and Akihiro Ishizu*
}

\begin{abstract}
From the Department of Medical Laboratory Science, * Faculty of Health Sciences, the Departments of Rheumatology, Endocrinology and Nephrology, ${ }^{\dagger}$ and Pathology," Faculty of Medicine and Graduate School of Medicine, and the Center for Cause of Death Investigation, "Graduate School of Medicine, Hokkaido University, Sapporo; the Department of Pathology, ${ }^{\ddagger}$ Sapporo City Hospital, Sapporo; the Research Division of Genome Companion Diagnostics, ${ }^{\S}$ Hokkaido University Hospital, Sapporo; and the Department of Dermatology,** St. Marianna University School of Medicine, Kawasaki, Japan
\end{abstract}

Accepted for publication

January 3, 2019.

Address correspondence to Akihiro Ishizu, M.D., Ph.D. Faculty of Health Sciences, Hokkaido University, Kita-12, Nishi-5, Kita-ku, Sapporo 0600812, Japan. E-mail: aishizu@ med.hokudai.ac.jp.

\begin{abstract}
Antineutrophil cytoplasmic antibody (ANCA)-associated vasculitis (AAV) is characterized by the production of ANCAs and systemic necrotizing vasculitis in small vessels. Disordered regulation of neutrophil extracellular traps (NETs) is critically involved in the pathogenesis of AAV. NETs are web-like DNA decorated with antimicrobial proteins; they are extruded from activated neutrophils. The principal degradation factor of NETs in vivo is DNase I; however, NETs resistant to DNase I can persist in tissues and can lead to the production of ANCAs. Deposition of NETs has been demonstrated in glomerular crescents and necrotizing vasculitis in AAV. Here, the amount of NETs in formalin-fixed, paraffinembedded tissue sections was examined, and the results for AAV were compared with the results for diseases that should be distinguished from AAV. NETs were more abundant in necrotizing vasculitis of AAV than in non-ANCA-associated vasculitis, or in granulomatous angiitis. Pulmonary granulomas in AAV and non-ANCA-associated diseases were further studied. The amount of NETs was significantly greater in necrotizing granulomas of AAV than in granulomas of sarcoidosis without necrosis. Although NETs were formed in necrotizing granulomas of tuberculosis equivalently to those formed in AAV, they were more susceptible to degradation by DNase I than were NETs in AAV. The formation and disordered degradation of NETs in necrotizing lesions are characteristics of AAV and are possibly related to its pathogenesis. (Am J Pathol 2019, 189: 839-846; https://doi.org/10.1016/j.ajpath.2019.01.007)
\end{abstract}

Antineutrophil cytoplasmic antibody (ANCA)-associated vasculitis (AAV) includes microscopic polyangiitis (MPA), granulomatosis with polyangiitis (GPA), and eosinophilic granulomatosis with polyangiitis (EGPA). ${ }^{1} \mathrm{AAV}$ is characterized by production of ANCAs and systemic necrotizing vasculitis in small vessels. In addition, necrotizing granulomas in the lung are typically observed in GPA. ANCAs are known as pathogenic autoantibodies in AAV, and the major target antigens are myeloperoxidase (MPO) and proteinase 3. ANCAs bind to the antigens and simultaneously bridge the antigens and bystander Fc $\gamma$ receptors on neutrophils primed by proinflammatory cytokines, such as tumor necrosis factor- $\alpha$. Consequently, the neutrophils are activated to release reactive oxygen species and lytic enzymes, in turn contributing to the development of small vessel vasculitis. This ANCA-cytokine sequence is pivotal for understanding the pathogenesis of $\mathrm{AAV}^{2}$

\footnotetext{
Supported by a Japan Research Committee of the Ministry of Health, Labour and Welfare for Intractable Vasculitis grant (A.I.) and Japan Agency for Medical Research and Development grant 15ek0109121 (A.I.). Disclosures: None declared.

Current address of T.K., Department of Dermatology, Tohoku Medical and Pharmaceutical University, Sendai, Japan.
} 
In 2004, Brinkmann et $\mathrm{al}^{3}$ reported that phorbol myristate acetate (PMA) stimulates neutrophils to extrude a decondensed DNA that forms an extracellular web-like structure decorated with antimicrobial proteins, including MPO, proteinase 3, and histones. These substances, termed neutrophil extracellular traps (NETs), can capture and kill microbes. Patients with chronic granulomatous disease who fail to generate NETs are susceptible to diverse bacterial and fungal infections. In contrast, restoration of NET formation in chronic granulomatous disease by gene therapy results in resistance to such infections. ${ }^{4}$ Thus, NET formation is an important event of innate immunity. However, some components of NETs, including MPO, proteinase 3, and histones, have adverse effects on the host, such as cytotoxicity to vascular endothelial cells and thrombogenicity. ${ }^{5}$

Kessenbrock et al ${ }^{6}$ were the first to report the relationship of AAV and NETs. They demonstrated the presence of NETs in glomerular crescents of AAV patients and that ANCAs could induce release of NETs from neutrophils primed by tumor necrosis factor- $\alpha$. It is currently considered that NETs extruded from activated neutrophils are implicated in vascular endothelial cell injury in the ANCA-cytokine sequence. ${ }^{7}$
Because of the adverse aspects to the host, NETs are strictly regulated in vivo. The most important NET degradation factor is serum DNase I. ${ }^{8}$ We have demonstrated that NETs resistant to degradation by DNase I are critically involved in the production of ANCAs, especially MPOANCAs. ${ }^{9}$ We have also demonstrated that sera of MPA patients have low DNase I activity and a correspondingly decreased ability to degrade NETs. ${ }^{10}$

PMA-stimulated neutrophils undergo cell death accompanied by NET formation. ${ }^{11}$ Because the characteristics of this type of cell death resemble neither typical necrosis nor apoptosis, Steinberg et $\mathrm{al}^{12}$ coined the term NETosis to describe neutrophilic cell death with NET formation. NETosis induced by PMA requires activation of the Raf-mitogen-activated protein extracellular signal-regulated kinase-extracellular signal-regulated kinase pathway and receptor-interacting serine/threonine-protein kinase 1-receptor-interacting serine/ threonine-protein kinase 3-mixed lineage kinase domain-like pseudokinase pathway. ${ }^{13,14}$ In this process, peptidylarginine deiminase 4-dependent citrullination of histones induces decondensation of DNA that results in a mixture of DNA and bactericidal proteins originally contained in intracytoplasmic

Table 1 Formalin-Fixed, Paraffin-Embedded Specimens Used in This Study

\begin{tabular}{|c|c|c|c|c|c|c|}
\hline Patient & Age, years & Sex & Diagnosis & Tissue obtained & Lesions, $n$ & Group in Figures 2 and 3 \\
\hline 1 & 71 & $\mathrm{~F}$ & MPA & Muscle biopsy & 1 & Necrotizing vasculitis in AAV \\
\hline 2 & 56 & M & MPA & Muscle biopsy & 4 & Necrotizing vasculitis in AAV \\
\hline 3 & 82 & $\mathrm{~F}$ & MPA & Kidney biopsy & 1 & Necrotizing vasculitis in AAV \\
\hline 4 & 61 & $\mathrm{~F}$ & EGPA & Intestine in autopsy & 2 & Necrotizing vasculitis in AAV \\
\hline 5 & 66 & M & GPA & Lung in autopsy & 3 & Necrotizing granulomas in AAV \\
\hline 6 & 58 & M & GPA & Lung resection & 1 & Necrotizing granulomas in AAV \\
\hline 9 & 78 & $\mathrm{~F}$ & PAN & Muscle biopsy & 2 & Necrotizing vasculitis in non-AAV \\
\hline 10 & 39 & M & PAN & Skin biopsy & 1 & Necrotizing vasculitis in non-AAV \\
\hline 11 & 45 & $\mathrm{~F}$ & PAN & Skin biopsy & 2 & Necrotizing vasculitis in non-AAV \\
\hline 12 & 27 & $\mathrm{~F}$ & CA & Skin biopsy & 1 & Necrotizing vasculitis in non-AAV \\
\hline 13 & 49 & $\mathrm{~F}$ & $\mathrm{CA}$ & Skin biopsy & 2 & Necrotizing vasculitis in non-AAV \\
\hline 18 & 71 & $\mathrm{~F}$ & GCA & Temporal artery biopsy & 1 & Granulomatous angiitis \\
\hline 19 & 57 & M & Tuberculosis & Lung resection & 1 & Necrotizing granulomas in non-ANCA-associated disease \\
\hline 20 & 75 & M & Tuberculosis & Lung resection & 2 & Necrotizing granulomas in non-ANCA-associated disease \\
\hline 21 & 73 & $\mathrm{~F}$ & Tuberculosis & Lung resection & 1 & Necrotizing granulomas in non-ANCA-associated disease \\
\hline 22 & 63 & M & Tuberculosis & Lung resection & 1 & Necrotizing granulomas in non-ANCA-associated disease \\
\hline 23 & 66 & M & Tuberculosis & Lung resection & 1 & Necrotizing granulomas in non-ANCA-associated disease \\
\hline 24 & 46 & $\mathrm{~F}$ & Sarcoidosis & Lung biopsy & 1 & Granulomas without necrosis in non-ANCA-associated disease \\
\hline 25 & 44 & M & Sarcoidosis & Lung biopsy & 2 & Granulomas without necrosis in non-ANCA-associated disease \\
\hline 26 & 63 & $\mathrm{~F}$ & Sarcoidosis & Lung resection & 1 & Granulomas without necrosis in non-ANCA-associated disease \\
\hline 27 & 45 & $\mathrm{~F}$ & Sarcoidosis & Lung resection & 1 & Granulomas without necrosis in non-ANCA-associated disease \\
\hline 28 & 36 & $\mathrm{~F}$ & Sarcoidosis & Lung resection & 1 & Granulomas without necrosis in non-ANCA-associated disease \\
\hline
\end{tabular}

F, female; M, male; AAV, ANCA-associated vasculitis; ANCA, antineutrophil cytoplasmic antibody; CA, cutaneous arteritis; EGPA, eosinophilic granulomatosis with polyangiitis; GCA, giant cell arteritis; GPA, granulomatosis with polyangiitis; MPA, microscopic polyangiitis; PAN, polyarteritis nodosa. 

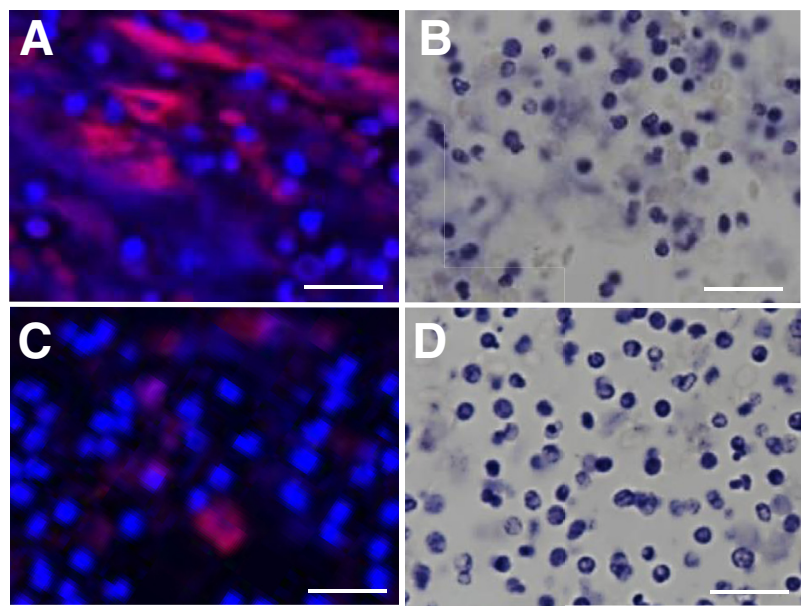

Figure 1 Staining of clots for visualization of neutrophil extracellular traps (NETs). Formalin-fixed, paraffin-embedded sections of clots of phorbol myristate acetate (PMA)-treated peripheral neutrophils were subjected to immunofluorescence staining for citrullinated histone 3 (Cit H3; red) (A and $\mathbf{C}$ ) and simple hematoxylin (SH) staining (B and $\mathbf{D})$. DAPI used for DNA staining is shown in blue. $\mathbf{A}$ and $\mathbf{B}$ : Before DNase I treatment. $\mathbf{C}$ and $\mathbf{D}$ : After DNase I treatment $\left(10 \mathrm{U} / \mathrm{mL}\right.$, for 1 hour, at $\left.37^{\circ} \mathrm{C}\right)$. Scale bars $=$ $20 \mu \mathrm{m}(\mathbf{A}-\mathbf{D})$.

granules. ${ }^{15}$ Thereafter, these substances are extruded from the ruptured plasma membrane of neutrophils. Thus, citrullinated histones can be reliable markers of NETs.

The presence of NETs has been shown in glomerular crescents, ${ }^{6}$ deep vein thromboses, ${ }^{16,17}$ and necrotizing vasculitis ${ }^{18}$ in AAV. Here, we examined the amounts of NETs in formalin-fixed, paraffin-embedded (FFPE) tissue sections and compared the results for AAV with the results for diseases that should be distinguished from AAV.

\section{Materials and Methods}

\section{Clots Containing NETs}

Human peripheral blood neutrophils were obtained from healthy volunteers by density centrifugation by using Polymorphprep (Axis-Shield, Dundee, Scotland). The neutrophils were suspended in RPMI 1640 medium that contained 5\% fetal bovine serum in a polypropylene tube $\left(1 \times 10^{6} / \mathrm{mL}\right)$ and subsequently exposed to $100 \mathrm{nmol} / \mathrm{L}$ PMA (Sigma-Aldrich, St. Louis, MO) for 4 hours at $37^{\circ} \mathrm{C}$. During incubation, the tube was periodically gently shaken. Thereafter, the samples were fixed with $10 \%$ formalin, and a clot was generated by the sodium alginate method. ${ }^{19}$ The clot was embedded in paraffin wax for making a FFPE specimen. This study was conducted with the permission of the Ethical Committee of the Faculty of Health Sciences, Hokkaido University (permission numbers 17-85 and 18-61).

\section{FFPE Tissue Sections}

Tissue samples from eight patients with AAV (three with MPA, four with GPA, and one with EGPA) were used. For controls, tissue samples from 10 patients with non-ANCAassociated vasculitis (three with polyarteritis nodosa, two with cutaneous arteritis, and five with giant cell arteritis), and 10 patients with non-vasculitic diseases (five with tuberculosis and five with sarcoidosis) were used. These samples were obtained by biopsy, surgery, or autopsy and were fixed with $10 \%$ formalin and embedded in paraffin wax. Details of the FFPE specimens used in this study are shown in Table 1.

\section{Immunofluorenscence Staining}

FFPE specimens were sliced into 4- $\mu \mathrm{m}$ sections and then deparaffinized with xylene. After antigen retrieval in an autoclave (at $121^{\circ} \mathrm{C}$ for 20 minutes) with Tris-EDTA buffer ( $\mathrm{pH} 9)$, the sections were soaked in Protein Block Serumfree (Dako, Glostrup, Denmark) for 10 minutes at room temperature to inhibit nonspecific binding of antibodies. Primary antibodies used were anti-citrullinated histone 3 (Cit H3; rabbit polyclonal; dilution 1:100; Abcam, Eugene, OR) and anti-CD15 (mouse IgM, ready-to-use; Dako). After incubation with primary antibodies for 1 hour at room temperature, the sections were washed with phosphatebuffered saline (PBS). Secondary antibodies used were Alexa Fluor 594-conjugated goat anti-rabbit IgG H\&L at 1:500 dilution (Abcam) and Alexa Fluor 488-conjugated goat anti-mouse IgM at 1:500 dilution (Invitrogen, Carlsbad, CA). After incubation with secondary antibodies for 1 hour at room temperature, the sections were washed with PBS and then mounted with a mounting solution that contained DAPI (Vector Laboratories, Burlingame, CA). The slides were observed under a fluorescence microscope.

\section{Hematoxylin Staining with or without Eosin Staining}

FFPE sections were deparaffinized with xylene and then allowed to react with hematoxylin solution. After washing the surplus stain out of the cytoplasm with $1 \%$ hydrochloric acid in $70 \%$ alcohol, the sections were allowed to react with or without eosin solution. The former is conventional hematoxylin and eosin (H\&E) staining. The latter was named simple hematoxylin (SH) staining.

\section{DNase I Treatment}

FFPE clot sections of PMA-treated neutrophils were deparaffinized with xylene and then subjected to antigen retrieval in an autoclave (at $121^{\circ} \mathrm{C}$ for 20 minutes) with citrate buffer ( $\mathrm{pH} \mathrm{6)}$. Thereafter, the sections were allowed to react with 1 or $10 \mathrm{U} / \mathrm{mL}$ DNase I (Invitrogen) for 1 hour at $37^{\circ} \mathrm{C}$. After washing with PBS, the sections were subjected to immunofluorescence staining for $\mathrm{Cit} \mathrm{H} 3$ and $\mathrm{SH}$ staining. FFPE tissue sections of AAV and tuberculosis were deparaffinized with xylene and then treated by DNase I similarly. After washing with PBS, the sections were mounted with the use of the DAPI-containing solution or 

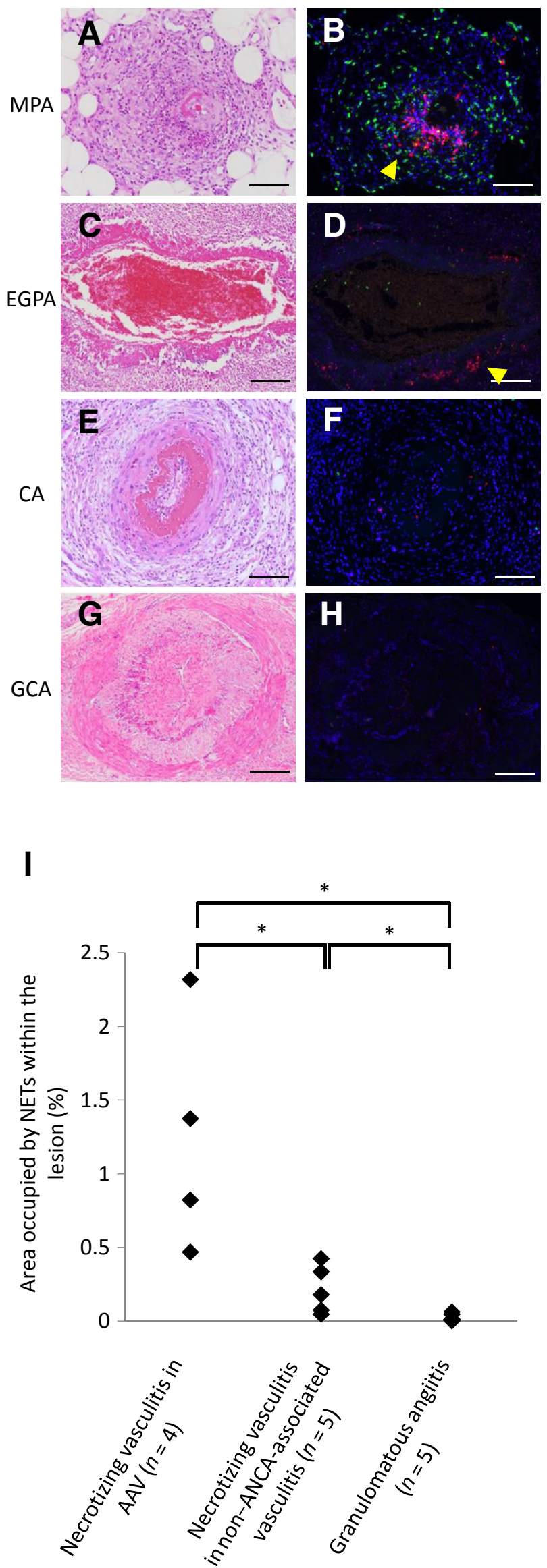

subjected to immunofluorescence staining for Cit H3/CD15 and SH staining.

\section{Image Analysis}

Image analysis was performed with ImageJ software version 1.51a (NIH, Bethesda, MD). The area occupied by NETs within the lesion (\%) was calculated as follows: [(area of NETs determined as Cit $\mathrm{H} 3$-positive area)/(lesion area determined by $H \& E$ staining) $] \times 100$. The percentage of NETs remaining after DNase I treatment [NETs residual rate (\%)] was calculated by using the mean brightness of the DAPI images or SH images as follows: [(mean brightness after DNase I treatment)/(mean brightness before DNase I treatment)] $\times 100$.

When multiple lesions were present in a specimen, the mean value of the area occupied by NETs within the lesion $(\%)$ and the NETs residual rate (\%) were calculated.

\section{Statistical Analysis}

The $U$-test was applied for comparison of two nonparametric groups. A $P$ value of $<0.05$ was regarded as statistically significant.

\section{Results}

\section{Visualization of NETs in FFPE Sections}

Immunofluorescence staining for Cit $\mathrm{H} 3$ is the method that has been generally applied to visualize NETs. ${ }^{20}$ Here, SH staining was additionally performed to observe NETs by light microscopy. Peripheral blood neutrophils stimulated by $100 \mathrm{nmol} / \mathrm{L}$ PMA were used for making FFPE clot specimens. Sections from the FFPE specimens were subjected to immunofluorescence staining for $\mathrm{Cit} \mathrm{H} 3$ and $\mathrm{SH}$ staining. In immunofluorescence staining, Cit $\mathrm{H} 3-$ positive DAPI-positive substances were determined as NETs (Figure 1A). From the finding that most of the Cit H3-positive area was also DAPI positive, the Cit $\mathrm{H} 3$ - positive area was regarded as the area of NETs for the remainder of this study. Because hematoxylin can stain nucleic acid, ${ }^{21} \mathrm{SH}$ staining was performed to visualize NETs in the FFPE sections. As expected, NETs were also identified as hematoxylin-stained substances around neutrophils (Figure 1B).

\footnotetext{
Figure 2 Presence of neutrophil extracellular traps (NETs) in vasculitic lesions. A-H: Formalin-fixed, paraffin-embedded tissue sections were subjected to hematoxylin and eosin staining $(\mathbf{A}, \mathbf{C}, \mathbf{E}$, and $\mathbf{G})$ and immunofluorescence staining for citrullinated histone 3 (Cit H3; red) and CD15 (green) (B, D, F, and $\mathbf{H})$. DNA is shown in blue. Arrowheads indicate NETs. I: Area occupied by NETs within the lesion, as determined by image analysis. ${ }^{*} P<0.05$. Scale bars: $100 \mu \mathrm{m}(\mathbf{A}, \mathbf{B}, \mathbf{E}$, and F); $200 \mu \mathrm{m}$ (C, D, G, and H). AAV, ANCA-associated vasculitis; ANCA, antineutrophil cytoplasmic antibody; CA, cutaneous arteritis; EGPA, eosinophilic granulomatosis with polyangiitis; GCA, giant cell arteritis; MPA, microscopic polyangiitis.
} 


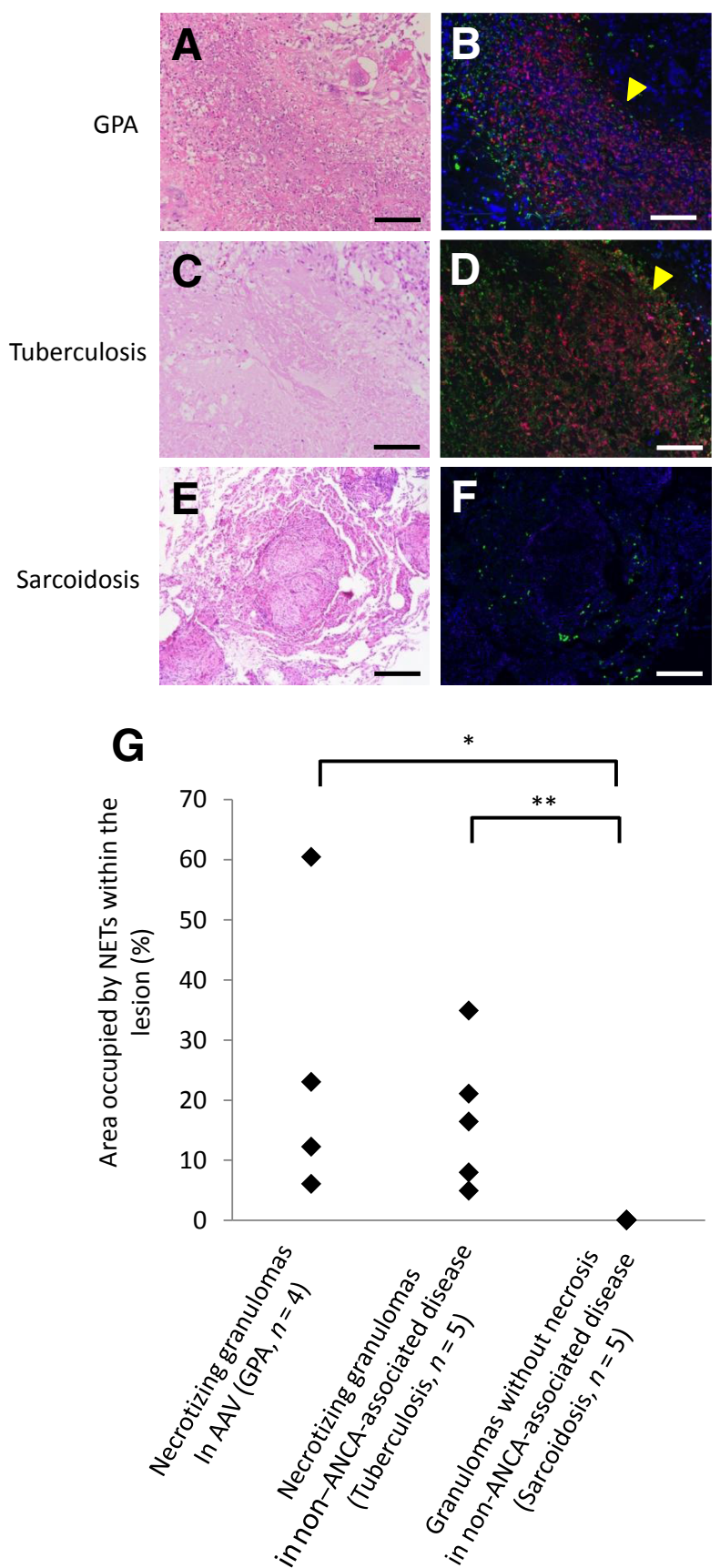

Figure 3 Presence of neutrophil extracellular traps (NETs) in pulmonary granulomas. A-F: Formalin-fixed, paraffin-embedded lung sections were subjected to hematoxylin and eosin staining (A, C, and E) and immunofluorescence staining for citrullinated histone 3 (Cit H3; red) and CD15 (B, D, and F; green). DNA is shown in blue. Arrowheads indicate NETs. G: Area occupied by NETs within the lesion, as determined by image analysis. ${ }^{*} P<0.05,{ }^{*} P<0.01$. Scale bars: $100 \mu \mathrm{m}$ (A, B, C, and D); $200 \mu \mathrm{m}$ (E and F). AAV, ANCA-associated vasculitis; ANCA, antineutrophil cytoplasmic antibody; GPA = granulomatosis with polyangiitis.

It has been demonstrated that NETs induced by PMA are digested by DNase I in the cell culture system. ${ }^{8}$ Thus, it was examined whether PMA-induced NETs in FFPE sections could also be digested by DNase I similarly to that under the cell culture conditions. The preliminary experiments indicated that $10 \mathrm{U} / \mathrm{mL}$ (but not $1 \mathrm{U} / \mathrm{mL}$ ) DNase I exposure for 1 hour at $37^{\circ} \mathrm{C}$ could effectively digest the PMA-induced NETs in FFPE sections. DNase I treatment $\left(10 \mathrm{U} / \mathrm{mL}\right.$, for 1 hour, at $\left.37^{\circ} \mathrm{C}\right)$ resulted in the digestion of NETs in FFPE sections (Figure 1, C and D). Cit $\mathrm{H} 3$ was not removed completely by the DNase I treatment, whereas almost all extracellular DNA stained by DAPI and hematoxylin was digested by DNase I.

\section{Presence of NETs in Vasculitis}

The vasculitides were divided into three groups - necrotizing vasculitis in AAV, necrotizing vasculitis in non-ANCAassociated vasculitis, and granulomatous angiitis-focusing on the presence of ANCAs and fibrinoid necrosis, and the amounts of NETs in each of the groups was then compared. $\mathrm{Cit} \mathrm{H} 3$-positive substances were present around fibrinoid necrosis in vasculitic lesions of AAV (Figure 2, A-D). The distribution of CD15-positive cells (regarded as neutrophils) was consistent with the localization of $\mathrm{Cit} \mathrm{H} 3$-positive substances in MPA but not in EGPA. A characteristic of EGPA was abundant infiltration of eosinophils around fibrinoid necrosis in vasculitic lesions. Because eosinophils could release extracellular traps, ${ }^{22}$ the $\mathrm{Cit} \mathrm{H} 3$ - positive substances in EGPA were thought to be derived from eosinophils.

Interestingly, the amount of NETs was much smaller in necrotizing vasculitis of non-ANCA-associated vasculitis (Figure 2, E and F). NETs were hardly detected in granulomatous angiitis of giant cell arteritis (Figure 2, G and H). The area occupied by NETs within the lesion was significantly greater in necrotizing vasculitis of AAV than in non-ANCA-associated vasculitis or granulomatous angiitis (Figure 2I). These findings were consistent with the concept that ANCA-induced NETs were involved in the pathogenesis of necrotizing vasculitis in AAV. ${ }^{7}$ It should be noted that the area occupied by NETs within the lesion (\%) varied among patients with AAV. This variance might be associated with the diverse phases of vasculitis usually observed in a patient with AAV. ${ }^{1}$

\section{Presence of NETs in Pulmonary Granulomas}

Next, the presence of NETs was determined in pulmonary granulomas. Pulmonary granulomas were divided into three groups - necrotizing granulomas in AAV, necrotizing granulomas in non-ANCA-associated diseases, and granulomas without necrosis in non-ANCA-associated diseases-focusing on the presence of ANCAs and necrosis. Abundant NETs were present in necrotizing granulomas in the lung tissues of patients with GPA (Figure 3, A and B) and tuberculosis (Figure 3, C and D) but not in pulmonary granulomas of sarcoidosis without necrosis (Figure 3, E and F). The distribution of CD15-positive cells (regarded as neutrophils) was consistent with the localization of NETs in both GPA and tuberculosis. The area occupied by NETs within the lesion was significantly greater in GPA and tuberculosis than in sarcoidosis (Figure 3G). 


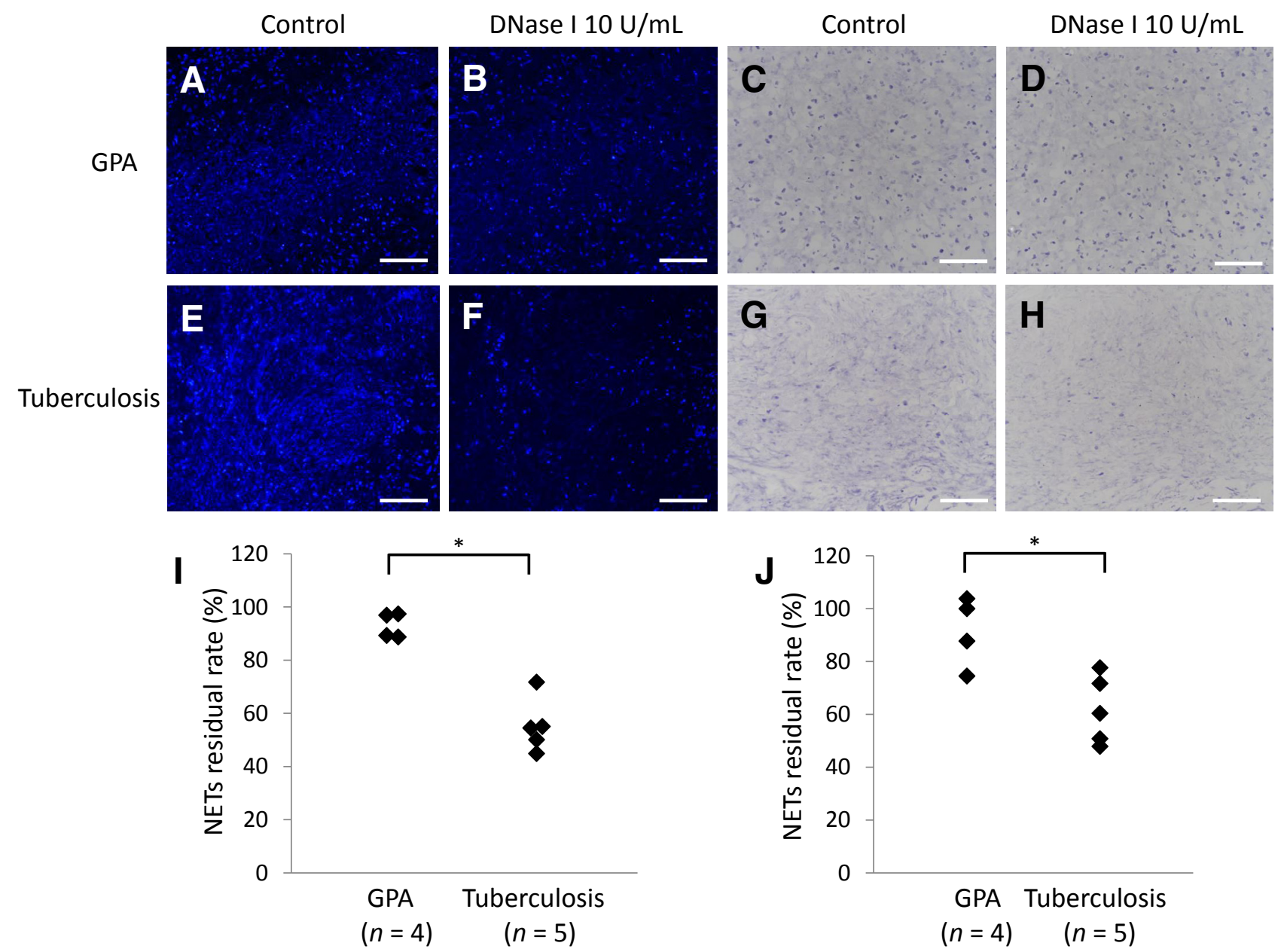

Figure 4 Disordered degradation of neutrophil extracellular traps (NETs) in granulomatosis with polyangiitis (GPA). Formalin-fixed, paraffin-embedded lung sections of GPA and tuberculosis were treated with or without $10 \mathrm{U} / \mathrm{mL}$ DNase I for 1 hour at $37^{\circ} \mathrm{C}$. A-H: After washing with phosphate-buffered saline, the sections were subjected to DAPI staining (A, B, E, and $\mathbf{F})$ or simple hematoxylin (SH) staining (C, D, G, and $\mathbf{H})$. A, C, E, and $\mathbf{G}$ : Before DNase I treatment. B, D, F, and $\mathbf{H}$ : After DNase I treatment. I: Percentage of NETs remaining after DNase I treatment (NETs residual rate), as determined by DAPI staining. J: NETs residual rate, as determined by $\mathrm{SH}$ staining. ${ }^{*} P<0.05$. Scale bars $=50 \mu \mathrm{m}(\mathbf{A}-\mathbf{H})$.

\section{Disordered Degradation of NETs in AAV}

NETs were present in necrotizing granulomas in the lung tissues of patients with GPA and tuberculosis. These findings were consistent because both ANCAs and mycobacterium infection can induce NETs. ${ }^{6,23,24}$ Because it was suspected that NETs resistant to DNase I were associated with ANCA production, ${ }^{16}$ DNase I resistance of NETs was examined in GPA and tuberculosis. FFPE lung tissue sections of GPA and tuberculosis treated with or without DNase I $(10 \mathrm{U} / \mathrm{mL}$, for 1 hour, at $37^{\circ} \mathrm{C}$ ) were subjected to DAPI staining and $\mathrm{SH}$ staining. Because NETs could be stained by a single dye in these methods, either method was expected to be suitable for evaluation of the degradation of NETs by DNase I. These results demonstrated that NETs in necrotizing granulomas in the lung tissues of GPA were not easily digested by DNase I compared with those of tuberculosis (Figure 4). On the contrary, Cit $\mathrm{H} 3$ remained even after the DNase I treatment in necrotizing granulomas of tuberculosis (Supplemental Figure S1). It was further examined if NETs in necrotizing

Table 2 NET Deposition in Vasculitic Lesions

\begin{tabular}{|c|c|c|c|}
\hline \multirow[b]{2}{*}{ Variable } & \multicolumn{2}{|l|}{ Necrotizing vasculitis } & \multirow[b]{2}{*}{ Granulomatous angiitis (GCA } \\
\hline & ANCA-associated (MPA, EGPA) & Non-ANCA-associated (PAN, CA) & \\
\hline NET deposition & + & - & - \\
\hline Resistance to DNase I & + & Not tested & Not tested \\
\hline
\end{tabular}

ANCA, antineutrophil cytoplasmic antibody; CA, cutaneous arteritis; EGPA, eosinophilic granulomatosis with polyangiitis; GCA, giant cell arteritis; MPA, microscopic polyangiitis; NET, neutrophil extracellular trap; PAN, polyarteritis nodosa. 
Table 3 NET Deposition in Necrotizing Granulomas

\begin{tabular}{llll}
\hline & Necrotizing granulomas & & \\
\cline { 2 - 4 } Variable & ANCA-associated (GPA) & Non-ANCA-associated (tuberculosis) & Granulomas without necrosis (sarcoidosis) \\
\hline NET deposition & + & + & - \\
Resistance to DNase I & + & - & Not tested \\
\hline
\end{tabular}

ANCA, antineutrophil cytoplasmic antibody; GPA, granulomatosis with polyangiitis; NET, neutrophil extracellular trap.

vasculitic lesions of MPA were digested by DNase I. The results suggested that they were also resistant to DNase I (Supplemental Figure S2).

\section{Discussion}

In this study, the following were demonstrated: i) NETs can be detected in FFPE sections by SH staining and by immunofluorescence staining for $\mathrm{Cit} \mathrm{H} 3$, and NETs in FFPE sections can be digested by DNase I in a manner similar to that under cell culture conditions; ii) NETs are present specifically in vasculitic lesions of AAV; and iii) DNase I-resistant NETs are present in necrotizing lesions of AAV (Tables 2 and 3).

Concerning the first issue, detection of NETs in human samples is still challenging. ${ }^{20}$ Currently, immunofluorescence staining for NET components, such as Cit H3, is regarded as the most reliable method for detecting NETs in tissue samples; however, this method requires working in the dark, which is somewhat of a burden for investigators. SH staining can be helpful in that specimens are observed under a light microscope. In addition, to the best of our knowledge, this study is the first to demonstrate that NETs in FFPE sections can be digested by DNase I treatment. Although DNA in the nuclei of cells in FFPE sections can also be digested by DNase I, extracellular DNA of NETs was more susceptible to the treatment $\left(10 \mathrm{U} / \mathrm{mL}\right.$ of DNase I, for 1 hour, at $\left.37^{\circ} \mathrm{C}\right)$ than was intranuclear DNA. The susceptibility to DNase I could be different for extracellular DNA and intranuclear DNA. As described below, DNase I resistance appears to be one of the characteristics of NETs in AAV. Because NETs themselves are formed in healthy individuals when necessary, the DNase I digestion test can be useful to distinguish ANCA-related NETs from non-ANCA-related NETs.

Second, it was determined that NETs were more abundant in necrotizing vasculitis of AAV than in non-ANCA-associated vasculitis, such as polyarteritis nodosa and cutaneous arteritis, or in granulomatous angiitis of giant cell arteritis. NETs were present around fibrinoid necrosis in the affected vessels. Yoshida et $\mathrm{al}^{18}$ also reported that Cit $\mathrm{H} 3$ was located around fibrinoid necrosis in necrotizing interlobular arteries of the kidney. Moreover, we have noted the presence of NETs around small vessels without fibrinoid necrosis in MPA patients (data not shown). Recent studies have demonstrated that ANCAinduced NETs possess cytotoxic activity toward vascular endothelial cells. ${ }^{25}$ Collectively, these findings suggest that NETs induced by ANCAs are responsible for the formation of fibrinoid necrosis in the vasculitic lesions of AAV.
Finally, it was demonstrated that NETs were present in necrotizing pulmonary granulomas of GPA and tuberculosis but not in pulmonary granulomas of sarcoidosis without necrosis. This is the first evidence of the presence of NETs in necrotizing granulomas of GPA. NETs were located in the necrotic lesions but not in granulation tissues surrounding the necrosis. This is inconsistent with the finding of vasculitic lesions; however, the reason has not been revealed. NETs are also present in necrotizing granulomas in the lungs of patients with tuberculosis. Mycobacterium tuberculosis is phagocytosed by neutrophils and alveolar macrophages, and the neutrophils release NETs to prevent the spread of $M$. tuberculosis. $^{24}$ Interestingly, NETs formed in the necrotizing granulomas of GPA were not easily degraded by DNase I compared with NETs formed in tuberculosis. We have demonstrated that DNase I-resistant NETs induced by the antithyroid drug propylthiouracil could induce the production of MPO-ANCAs and subsequent development of AAV in vivo. ${ }^{9}$ These findings collectively suggest that DNase $\mathrm{I}-$ resistant NETs in necrotizing granulomas of GPA can be a source of ANCA antigens. The mechanism underlying how NETs acquire resistance to DNase I in AAV cannot be currently demonstrated. However, this issue seems to be important; thus, a comparative transcriptome analysis between NETs in AAV and in non-ANCA-associated vasculitis is now being performed.

\section{Conclusions}

In conclusion, the collective findings suggest that the formation and disordered degradation of NETs in necrotizing lesions are characteristics of AAV and are possibly related to its pathogenesis.

\section{Acknowledgments}

S.M., S.I., T.T., Y.H., U.T., T.K., and A.I. designed the study; S.M., M.N., E.F., and Y.N. performed the experiments; S.M., D.N., S.T., U.T., T.A., and A.I. analyzed the data; S.M., U.T., and A.I. wrote the manuscript.

\section{Supplemental Data}

Supplemental material for this article can be found at https://doi.org/10.1016/j.ajpath.2019.01.007. 


\section{References}

1. Jennette JC, Falk RJ, Bacon PA, Basu N, Cid MC, Ferrario F, FloresSuarez LF, Gross WL, Guillevin L, Hagen EC, Hoffman GS, Jayne DR, Kallenberg CG, Lamprecht P, Langford CA, Luqmani RA, Mahr AD, Matteson EL, Merkel PA, Ozen S, Pusey CD, Rasmussen N, Rees AJ, Scott DG, Specks U, Stone JH, Takahashi K, Watts RA: 2012 revised International Chapel Hill Consensus Conference Nomenclature of Vasculitides. Arthritis Rheum 2013, 65:1-11

2. Csernok E: Anti-neutrophil cytoplasmic antibodies and pathogenesis of small vessel vasculitides. Autoimmun Rev 2003, 2:158-164

3. Brinkmann V, Reichard U, Goosmann C, Fauler B, Uhlemann Y, Weiss DS, Weinrauch Y, Zychlinsky A: Neutrophil extracellular traps kill bacteria. Science 2004, 303:1532-1535

4. Bianchi M, Hakkim A, Brinkmann V, Siler U, Seger RA, Zychlinsky A, Reichenbach J: Restoration of NET formation by gene therapy in CGD controls aspergillosis. Blood 2009, 114:2619-2622

5. Doring Y, Weber C, Soehnlein O: Footprints of neutrophil extracellular traps as predictors of cardiovascular risk. Arterioscler Thromb Vasc Biol 2013, 33:1735-1736

6. Kessenbrock K, Krumbholz M, Schonermarck U, Back W, Gross WL, Werb Z, Grone HJ, Brinkmann V, Jenne DE: Netting neutrophils in autoimmune small-vessel vasculitis. Nat Med 2009, 15:623-625

7. Jennette JC, Falk RJ: Pathogenesis of antineutrophil cytoplasmic autoantibody-mediated disease. Nat Rev Rheumatol 2014, 10:463-473

8. Hakkim A, Furnrohr BG, Amann K, Laube B, Abed UA, Brinkmann V, Herrmann M, Voll RE, Zychlinsky A: Impairment of neutrophil extracellular trap degradation is associated with lupus nephritis. Proc Natl Acad Sci U S A 2010, 107:9813-9818

9. Nakazawa D, Tomaru U, Suzuki A, Masuda S, Hasegawa R, Kobayashi T, Nishio S, Kasahara M, Ishizu A: Abnormal conformation and impaired degradation of propylthiouracil-induced neutrophil extracellular traps: implications of disordered neutrophil extracellular traps in a rat model of myeloperoxidase antineutrophil cytoplasmic antibody-associated vasculitis. Arthritis Rheum 2012, 64:3779-3787

10. Nakazawa D, Shida H, Tomaru U, Yoshida M, Nishio S, Atsumi T, Ishizu A: Enhanced formation and disordered regulation of NETs in myeloperoxidase-ANCA-associated microscopic polyangiitis. J Am Soc Nephrol 2014, 25:990-997

11. Fuchs TA, Abed U, Goosmann C, Hurwitz R, Schulze I, Wahn V, Weinrauch Y, Brinkmann V, Zychlinsky A: Novel cell death program leads to neutrophil extracellular traps. J Cell Biol 2007, 176:231-241

12. Steinberg BE, Grinstein S: Unconventional roles of the NADPH oxidase: signaling, ion homeostasis, and cell death. Sci STKE 2007, 2007:pe11

13. Hakkim A, Fuchs TA, Martinez NE, Hess S, Prinz H, Zychlinsky A, Waldmann H: Activation of the Raf-MEK-ERK pathway is required for neutrophil extracellular trap formation. Nat Chem Biol 2011, 7: 75-77

14. Desai J, Kumar SV, Mulay SR, Konrad L, Romoli S, Schauer C, Herrmann M, Bilyy R, Muller S, Popper B, Nakazawa D, Weidenbusch M, Thomasova D, Krautwald S, Linkermann A, Anders HJ: PMA and crystal-induced neutrophil extracellular trap formation involves RIPK1-RIPK3-MLKL signaling. Eur J Immunol 2016, 46:223-229

15. Li P, Li M, Lindberg MR, Kennett MJ, Xiong N, Wang Y: PAD4 is essential for antibacterial innate immunity mediated by neutrophil extracellular traps. J Exp Med 2010, 207:1853-1862

16. Nakazawa D, Tomaru U, Yamamoto C, Jodo S, Ishizu A: Abundant neutrophil extracellular traps in thrombus of patient with microscopic polyangiitis. Front Immunol 2012, 3:333

17. Imamoto T, Nakazawa D, Shida H, Suzuki A, Otsuka N, Tomaru U, Ishizu A: Possible linkage between microscopic polyangiitis and thrombosis via neutrophil extracellular traps. Clin Exp Rheumatol 2014, 32:149-150

18. Yoshida M, Sasaki M, Sugisaki K, Yamaguchi Y, Yamada M: Neutrophil extracellular trap components in fibrinoid necrosis of the kidney with myeloperoxidase-ANCA-associated vasculitis. Clin Kidney J 2013, 6:308-312

19. Yang SH, Wu CC, Shih TT, Chen PQ, Lin FH: Three-dimensional culture of human nucleus pulposus cells in fibrin clot: comparisons on cellular proliferation and matrix synthesis with cells in alginate. Artif Organs 2008, 32:70-73

20. Masuda S, Nakazawa D, Shida H, Miyoshi A, Kusunoki Y, Tomaru U, Ishizu A: NETosis markers: quest for specific, objective, and quantitative markers. Clin Chim Acta 2016, 459:89-93

21. Kiernan JA: Does progressive nuclear staining with hemalum (alum hematoxylin) involve DNA, and what is the nature of the dyechromatin complex? Biotech Histochem 2018, 93:133-148

22. Ueki S, Tokunaga T, Fujieda S, Honda K, Hirokawa M, Spencer LA, Weller PF: Eosinophil ETosis and DNA traps: a new look at eosinophilic inflammation. Curr Allergy Asthma Rep 2016, 16:54

23. Nakazawa D, Shida H, Kusunoki Y, Miyoshi A, Nishio S, Tomaru U, Atsumi T, Ishizu A: The responses of macrophages in interaction with neutrophils that undergo NETosis. J Autoimmun 2016, 67:19-28

24. Ramos-Kichik V, Mondragon-Flores R, Mondragon-Castelan M, Gonzalez-Pozos S, Muniz-Hernandez S, Rojas-Espinosa O, ChaconSalinas R, Estrada-Parra S, Estrada-Garcia I: Neutrophil extracellular traps are induced by Mycobacterium tuberculosis. Tuberculosis (Edinb) 2009, 89:29-37

25. Schreiber A, Rousselle A, Becker JU, von Massenhausen A, Linkermann A, Kettritz R: Necroptosis controls NET generation and mediates complement activation, endothelial damage, and autoimmune vasculitis. Proc Natl Acad Sci U S A 2017, 114:E9618-E9625 